\title{
The Electric Field Outside and Inside a Resistive Spherical Shell Carrying a Steady Azimuthal Current
}

\author{
J. A. Hernandes ${ }^{*}$ and A. K. T. Assis ${ }^{\dagger}$ \\ Instituto de Física ‘Gleb Wataghin’ Universidade Estadual de Campinas - Unicamp 13083-970 Campinas, São Paulo, Brasil
}

Received September 9, 2004; revised version received February 15, 2005; accepted February 18, 2005

PACS number: $41.20 . \mathrm{CV}$

\begin{abstract}
We calculate the potential, electric field and surface charges outside and inside a resistive spherical shell carrying a steady azimuthal current. We obtain a timeindependent electric field different from zero in both regions.

\section{Introduction}

In the past few years there has been a renewed interest in the electric field outside and inside ohmic conductors carrying steady currents. The analytical cases known in the literature include coaxial cables, [1, pp. 125-130], [2, pp. 318 and 509-511], [3], [4], [5, pp. 336-337] and [6]; a long solenoid with azimuthal current, [2, p. 318] and [7]; transmission lines, [8, p. 262] and more detailed in [9]; a long straight wire, [10]; conductor plates carrying steady currents, [11]; resistive strips of finite width, [12]; and a toroidal conductor carrying a steady current, [13].

Our goal in this work is to consider a steady azimuthal current flowing in a resistive spherical shell. The mathematical difficulty is at an intermediate degree between the infinite cylindrical shell, [7], and the toroidal conductor, [13]. The importance of the present case is that we can obtain exact analytical solutions for the external and internal distribution of surface charges, potential and electric field which are not so complex as in the toroidal conductor. Despite this fact they show clearly the existence of an electric field outside a resistive conductor bounded in a finite volume of space.
\end{abstract}

\section{Description of the problem}

Consider a resistive spherical shell of radius $a$, centered at the origin. We suppose an idealized linear battery located along a meridian of the shell (like Greenwich Meridian) and maintaining a constant electromotive force (emf) between its left and right sides. That is, the battery is a semi-circle in the plane $y=0$ with its extremities at $(x, y, z)=(0,0, \pm a)$ and its central point at $(x, y, z)=(-a, 0,0)$. Utilizing spherical coordinates $(r, \theta, \varphi)$ the linear battery is then located at $(a, \theta, \pi)$. We suppose that the emf generated by the battery does not depend upon the polar angle $\theta$. The battery generates a steady current flowing along the shell in the clockwise direction $(-\hat{\varphi})$, see Figs. 1 and 2 . The medium inside and outside the spherical shell is supposed to be air or vacuum. According to Ohm's law, the potential $\phi$ along the surface is given by:

$\phi(a, \theta, \varphi)=\phi_{A}+\phi_{B} \frac{\varphi}{2 \pi}$.

\footnotetext{
*Elecronic address: julioher@ifi.unicamp.br

†Electronic address: assis@ifi.unicamp.br; URL: http://www.ifi.unicamp. $\mathrm{br} / \sim$ assis
}

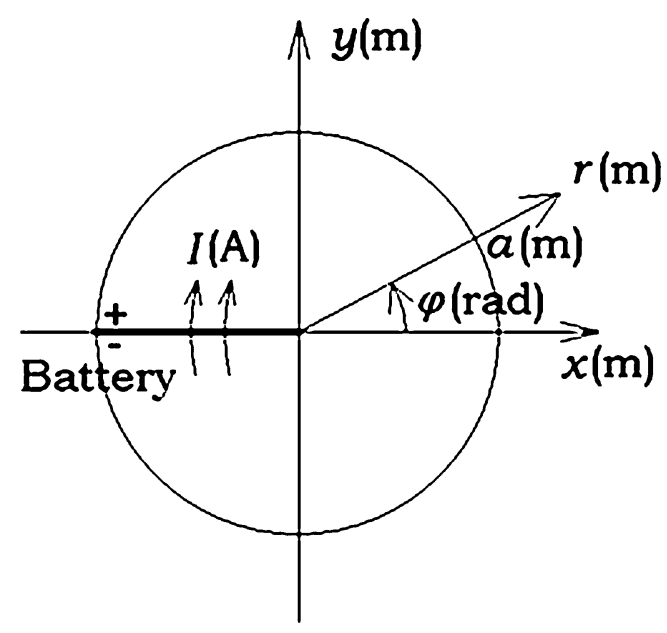

Fig. 1. Geometry of the resistive spherical shell with radius $a$ projected in the plane $z=0$. Notice that the semi-circular battery, represented by the bold line, is seen as a straight line for $-a \leq x \leq 0$. The idealized linear battery located at the spherical coordinates $(r, \theta, \varphi)=(a, \theta, \pi)$ generates a steady current $I$ (A) flowing along the surface of the shell in the clockwise direction $(-\hat{\varphi})$.

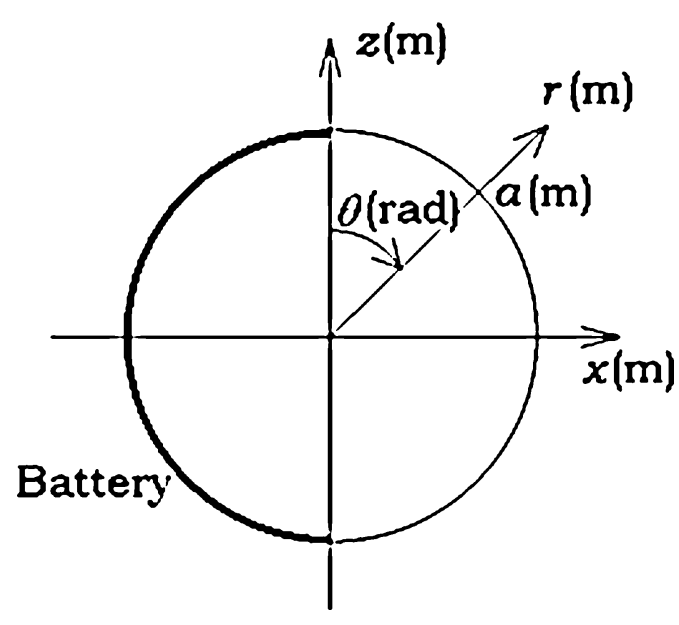

Fig. 2. Projection of the spherical shell in the plane $y=0$. The bold line represents the semi-circular battery.

The goal is to find solutions of Laplace's equation $\nabla^{2} \phi=0$ outside and inside the spherical shell utilizing Eq. (1) as a boundary condition, together with finite values of the potential at the center of the shell and at infinity. The electric field is then found by $\vec{E}=-\nabla \phi$. Lastly the surface charge density $\sigma$ is obtained by the standard procedure of taking the radial components of the external and internal electric fields when $r \rightarrow a$. 


\section{General solution}

Laplace's equation in spherical coordinates can be written as:

$\nabla^{2} \phi=\frac{\partial^{2} \phi}{\partial r^{2}}+\frac{2}{r} \frac{\partial \phi}{\partial r}+\frac{1}{r^{2}} \frac{\partial^{2} \phi}{\partial \theta^{2}}+\frac{\cot \theta}{r^{2}} \frac{\partial \phi}{\partial \theta}+\frac{1}{r^{2} \sin ^{2} \theta} \frac{\partial^{2} \phi}{\partial \varphi^{2}}=0$.

The electric potential $\phi$ can be solved utilizing the method of separation of variables, $\phi(r, \theta, \varphi)=R(r) \Theta(\theta) \Phi(\varphi)$. This yields the following equations for the functions $R, \Theta$ and $\Phi$, [14, pp. 24-27]:

$R^{\prime \prime}+\frac{2}{r} R^{\prime}-\frac{\alpha_{2}}{r^{2}} R=0$,

$\Theta^{\prime \prime}+\Theta^{\prime} \cot \theta+\left(\alpha_{2}-\frac{\alpha_{1}}{\sin ^{2} \theta}\right) \Theta=0$,

$\Phi^{\prime \prime}+\alpha_{1} \Phi=0$

where $\alpha_{1}$ and $\alpha_{2}$ are constants. The function $\Phi(\varphi)$ must be periodic in $\varphi$, that is, $\Phi(0)=\Phi(2 \pi)$. This implies $\alpha_{1}=q^{2}$, where $q=0,1,2, \ldots$ The solutions of Eq. (5) are then $\Phi_{q}^{(1)}=$ $\sin (q \varphi)$ and $\Phi_{q}^{(2)}=\cos (q \varphi)$. Eq. (4) is the associated Legendre' equation, [15, sec. 12.5]. In order to have finite solutions at $\theta=0 \mathrm{rad}$ and at $\theta=\pi \mathrm{rad}$ the constant $\alpha_{2}$ must have the form $\alpha_{2}=p(p+1)$, with $p=0,1,2, \ldots$ The solutions of Eq. (4) are then the associated Legendre functions of first and second kind, namely, $\Theta_{p q}^{(1)}=P_{p}^{q}(\cos \theta)$ and $\Theta_{p q}^{(2)}=Q_{p}^{q}(\cos \theta)$. When $q=0$ they reduce to Legendre polynomial, $P_{p}(\cos \theta)$, and to Legendre function of the second kind, $Q_{p}(\cos \theta)$, respectively. The solutions of Eq. (3) with $\alpha_{2}=p(p+1)$ are given by $R_{p}^{(1)}=r^{p}$ and $R_{p}^{(2)}=r^{-p-1}$.

The potential must remain finite at every point in space. The solution $R_{p}^{(1)}=r^{p}$ diverges when $r \rightarrow \infty$ and $p \geq 1$. For this reason we eliminate it outside the shell. By imposing that the potential goes to zero when $r \rightarrow \infty$ we can also eliminate the solution with $p=0$. Analogously we eliminate the solution $R_{p}^{(2)}=r^{-p-1}$ inside the shell as it diverges when $r \rightarrow 0$. The function $P_{p}^{q}(\cos \theta)$ is finite for $0 \mathrm{rad} \leq \theta \leq \pi \mathrm{rad}$. On the other hand, $Q_{p}^{q}(\cos \theta)$ diverges at $\theta=0 \mathrm{rad}$ and at $\theta=\pi \mathrm{rad}$. We then eliminate it both inside and outside the shell. The finite solutions for the potential outside and inside the shell are then given by the combination of all possible values of $R_{p}(r), \Theta_{p q}(\theta)$ and $\Phi_{q}(\varphi)$, respectively:

$$
\begin{aligned}
\phi_{o}(r \geq a, \theta, \varphi)= & \sum_{p=0}^{\infty} r^{-(p+1)}\left\{A_{p} P_{p}(\cos \theta)+\sum_{q=1}^{\infty}\left[B_{p q} \sin (q \varphi)\right.\right. \\
& \left.\left.+C_{p q} \cos (q \varphi)\right] P_{p}^{q}(\cos \theta)\right\}, \\
\phi_{i}(r \leq a, \theta, \varphi)= & \sum_{p=0}^{\infty} r^{p}\left\{D_{p} P_{p}(\cos \theta)+\sum_{q=1}^{\infty}\left[E_{p q} \sin (q \varphi)\right.\right. \\
& \left.\left.+F_{p q} \cos (q \varphi)\right] P_{p}^{q}(\cos \theta)\right\} .
\end{aligned}
$$

In order to obtain the coefficients $A_{p}, B_{p q}, C_{p q}, D_{p}, E_{p q}$ and $F_{p q}$ we must apply the boundary condition at the surface of the shell, $r=a$. Expanding Eq. (1) in Fourier series, [7]:

$$
\phi(a, \theta, \varphi)=\phi_{A}+\phi_{B} \frac{\varphi}{2 \pi}=\phi_{A}+\frac{\phi_{B}}{\pi}\left[\sum_{q=1}^{\infty} \frac{(-1)^{q-1}}{q} \sin (q \varphi)\right] \text {. }
$$

As there are no terms in $\cos (q \varphi)$ in Eq. (8) we obtain immediately $C_{p q}=F_{p q}=0$.

First we find the coefficients $A_{p}$ and $B_{p q}$ for the region outside the shell $(r \geq a)$. Eq. (6) calculated at $r=a$ combined with Eq. (8) yields the following equations:

$$
\begin{aligned}
& \phi_{A}=\sum_{p=0}^{\infty} a^{-(p+1)} A_{p} P_{p}(\cos \theta), \\
& \frac{\phi_{B}}{\pi} \frac{(-1)^{q-1}}{q}=\sum_{p=0}^{\infty} a^{-(p+1)} B_{p q} P_{p}^{q}(\cos \theta) .
\end{aligned}
$$

To find the coefficients $A_{p}$ and $B_{p q}$ we multiply both sides of Eq. (9) by $P_{\ell}(\cos \theta) \sin \theta \mathrm{d} \theta$, both sides of Eq. (10) by $P_{\ell}^{q}(\cos \theta) \sin \theta \mathrm{d} \theta$, and integrate from $0 \mathrm{rad}$ to $\pi \mathrm{rad}$. We then utilize the orthogonality relation of Legendre polynomials, [15, Eq. (12.104)]:

$\int_{0}^{\pi} P_{p}^{q}(\cos \theta) P_{\ell}^{q}(\cos \theta) \sin \theta \mathrm{d} \theta=\frac{2}{2 p+1} \frac{(p+q) !}{(p-q) !} \delta_{p \ell}$,

where $\delta_{p \ell}$ is the Kronecker delta function, which is 1 for $p=q$, and 0 for $p \neq q$. This yields:

$A_{p}=a \phi_{A} \delta_{p 0}$

and

$B_{p q}=\frac{\phi_{B}}{\pi} a^{p+1} \frac{(-1)^{q-1}}{q} \frac{2 p+1}{2} \frac{(p-q) !}{(p+q) !} I_{p q}$,

where we defined:

$I_{p q} \equiv \int_{0}^{\pi} P_{p}^{q}(\cos \theta) \sin \theta \mathrm{d} \theta$

Notice that $I_{p q}=0$ for $p+q$ odd due to the parity property of the associated Legendre functions, [15, p. 725]. Recurrence formulas can be used to evaluate the integrals in $I_{p q},[16]$.

We can change the upper limit of the summation over $q$ in Eq. (6) from $\infty$ to $p$, because $P_{p}^{q}(\xi)=0$ for $q>p$. The final solution for the potential outside a spherical shell conducting a steady azimuthal current is given by:

$$
\begin{aligned}
\phi_{o}(r \geq a, \theta, \varphi)= & \phi_{A} \frac{a}{r}+\frac{\phi_{B}}{2 \pi}\left[\sum_{p=1}^{\infty} \sum_{q=1}^{p} \frac{a^{p+1}}{r^{p+1}} \frac{(-1)^{q-1}}{q}(2 p+1)\right. \\
& \left.\times \frac{(p-q) !}{(p+q) !} I_{p q} P_{p}^{q}(\cos \theta) \sin (q \varphi)\right]
\end{aligned}
$$

It is useful to keep in mind that the summation order can be inverted, from $\sum_{p=1}^{\infty} \sum_{q=1}^{p}$ to $\sum_{q=1}^{\infty} \sum_{p=q}^{\infty}$.

For the region far from the origin, $r \gg a$, the two most relevant terms of Eq. (15) are:

$\phi_{o}(r \gg a, \theta, \varphi) \approx \phi_{A} \frac{a}{r}+\phi_{B} \frac{3 a^{2}}{8 r^{2}} \sin \theta \sin \varphi$.

This can be understood as the potential of a point charge $q_{\text {sphere }}=4 \pi \varepsilon_{0} \phi_{A} a$ at the center of the shell plus the potential of 
an electric dipole of moment $\vec{p}_{\text {sphere }}$ located at the origin with $\vec{p}_{\text {sphere }}=\left(3 \pi \varepsilon_{0} \phi_{B} a^{2} / 2\right) \hat{y}$, namely:

$\phi_{o}(r \gg a, \theta, \varphi)=\frac{q_{\text {sphere }}}{4 \pi \varepsilon_{0} r}+\frac{\vec{p}_{\text {sphere }} \cdot \vec{r}}{4 \pi \varepsilon_{0} r^{3}}$.

Here $\varepsilon_{0}=8.85 \times 10^{-12} \mathrm{C}^{2} \mathrm{~N}^{-1} \mathrm{~m}^{-2}$ is the permittivity of free space.

The solution for the potential inside the sphere $(r \leq a), \phi_{i}$, can be found by changing $(a / r)^{p+1} \rightarrow(r / a)^{p},[17$, p. 91$]$ :

$$
\begin{aligned}
\phi_{i}(r \leq a, \theta, \varphi)= & \phi_{A}+\frac{\phi_{B}}{2 \pi}\left[\sum_{p=1}^{\infty} \sum_{q=1}^{p} \frac{r^{p}}{a^{p}} \frac{(-1)^{q-1}}{q}(2 p+1)\right. \\
& \left.\times \frac{(p-q) !}{(p+q) !} I_{p q} P_{p}^{q}(\cos \theta) \sin (q \varphi)\right],
\end{aligned}
$$

where $I_{p q}$ is given by Eq. (14).

Utilizing that (as can be seen multiplying both sides of Eq. (19) by $P_{\ell}^{q}(\cos \theta) \sin \theta \mathrm{d} \theta$, integrating from $\theta=0 \operatorname{rad}$ to $\theta=\pi \mathrm{rad}$ and finally applying Eqs. (14), (13) and (8)):

$\sum_{p=1}^{\infty} \sum_{q=1}^{p} \frac{(-1)^{q-1}}{q}(2 p+1) \frac{(p-q) !}{(p+q) !} I_{p q} P_{p}^{q}(\cos \theta) \sin (q \varphi)=\varphi$,

we obtain from Eqs. (15) and (18) in the limit $r \rightarrow a$ that $\phi_{o}(a, \theta, \varphi)=\phi_{i}(a, \theta, \varphi)=\phi_{A}+\phi_{B} \varphi / 2 \pi$, as expected.

\section{Electric field and surface charges}

The electric field in spherical coordinates is given by:

$\vec{E}=-\nabla \phi=-\frac{\partial \phi}{\partial r} \hat{r}-\frac{1}{r} \frac{\partial \phi}{\partial \theta} \hat{\theta}-\frac{1}{r \sin \theta} \frac{\partial \phi}{\partial \varphi} \hat{\varphi}$.

This yields the following components outside and inside the shell, respectively:

$$
\begin{aligned}
& E_{r, o}=\phi_{A} \frac{a}{r^{2}}+\frac{\phi_{B}}{2 \pi}\left[\sum_{p=1}^{\infty} \sum_{q=1}^{p} \frac{a^{p+1}}{r^{p+2}} \frac{(-1)^{q-1}}{q}(p+1)(2 p+1)\right. \\
& \left.\times \frac{(p-q) !}{(p+q) !} I_{p q} P_{p}^{q}(\cos \theta) \sin (q \varphi)\right] \text {, } \\
& E_{\theta, o}=\frac{\phi_{B}}{2 \pi}\left[\sum_{p=1}^{\infty} \sum_{q=1}^{p} \frac{a^{p+1}}{r^{p+2}} \frac{(-1)^{q-1}}{q}(2 p+1)\right. \\
& \left.\times \frac{(p-q) !}{(p+q) !} I_{p q} P_{p}^{q^{\prime}}(\cos \theta) \sin \theta \sin (q \varphi)\right], \\
& E_{\varphi, o}=-\frac{\phi_{B}}{2 \pi}\left[\sum_{p=1}^{\infty} \sum_{q=1}^{p} \frac{a^{p+1}}{r^{p+2}}(-1)^{q-1}(2 p+1)\right. \\
& \left.\times \frac{(p-q) !}{(p+q) !} I_{p q} \frac{P_{p}^{q}(\cos \theta)}{\sin \theta} \cos (q \varphi)\right], \\
& E_{r, i}=-\frac{\phi_{B}}{2 \pi}\left[\sum_{p=1}^{\infty} \sum_{q=1}^{p} \frac{r^{p-1}}{a^{p}} \frac{(-1)^{q-1}}{q} p(2 p+1)\right. \\
& \left.\times \frac{(p-q) !}{(p+q) !} I_{p q} P_{p}^{q}(\cos \theta) \sin (q \varphi)\right],
\end{aligned}
$$

$$
\begin{aligned}
E_{\theta, i}= & \frac{\phi_{B}}{2 \pi}\left[\sum_{p=1}^{\infty} \sum_{q=1}^{p} \frac{r^{p-1}}{a^{p}} \frac{(-1)^{q-1}}{q}(2 p+1)\right. \\
& \left.\times \frac{(p-q) !}{(p+q) !} I_{p q} P_{p}^{q^{\prime}}(\cos \theta) \sin \theta \sin (q \varphi)\right], \\
E_{\varphi, i}= & -\frac{\phi_{B}}{2 \pi}\left[\sum_{p=1}^{\infty} \sum_{q=1}^{p} \frac{r^{p-1}}{a^{p}}(-1)^{q-1}(2 p+1)\right. \\
& \left.\times \frac{(p-q) !}{(p+q) !} I_{p q} \frac{P_{p}^{q}(\cos \theta)}{\sin \theta} \cos (q \varphi)\right] .
\end{aligned}
$$

In Eqs. (22) and (25) $P_{p}^{q^{\prime}}(\xi)$ is the derivative of the associated Legendre function $P_{p}^{q}(\xi)$ relative to its argument $\xi$.

From Eqs. (22), (25) and (19) we obtain in the limit $r \rightarrow a$ that:

$$
\begin{aligned}
E_{\theta, o}(a, \theta, \varphi)= & E_{\theta, i}(a, \theta, \varphi) \\
= & \frac{\phi_{B}}{2 \pi a}\left[\sum_{p=1}^{\infty} \sum_{q=1}^{p} \frac{(-1)^{q-1}}{q}(2 p+1)\right. \\
& \left.\times \frac{(p-q) !}{(p+q) !} I_{p q} P_{p}^{q^{\prime}}(\cos \theta) \sin \theta \sin (q \varphi)\right] \\
= & \frac{\phi_{B}}{2 \pi a} \frac{\mathrm{d}}{\mathrm{d} \theta}\left[\sum_{p=1}^{\infty} \sum_{q=1}^{p} \frac{(-1)^{q-1}}{q}(2 p+1) \frac{(p-q) !}{(p+q) !}\right. \\
& \left.\times I_{p q} P_{p}^{q}(\cos \theta) \sin (q \varphi)\right] \\
= & \frac{\phi_{B}}{2 \pi a} \frac{\mathrm{d}}{\mathrm{d} \theta} \varphi=0 .
\end{aligned}
$$

From Eqs. (23), (26) and (19) we obtain in the limit $r=a$ that:

$$
\begin{aligned}
E_{\varphi, o}(a, \theta, \varphi)= & E_{\varphi, i}(a, \theta, \varphi) \\
= & -\frac{\phi_{B}}{2 \pi a \sin \theta}\left[\sum_{p=1}^{\infty} \sum_{q=1}^{p}(-1)^{q-1}(2 p+1) \frac{(p-q) !}{(p+q) !}\right. \\
& \left.\times I_{p q} P_{p}^{q}(\cos \theta) \cos (q \varphi)\right] \\
= & -\frac{\phi_{B}}{2 \pi a \sin \theta} \frac{\mathrm{d}}{\mathrm{d} \varphi}\left[\sum_{p=1}^{\infty} \sum_{q=1}^{p} \frac{(-1)^{q-1}}{q}(2 p+1)\right. \\
& \left.\times \frac{(p-q) !}{(p+q) !} I_{p q} P_{p}^{q}(\cos \theta) \sin (q \varphi)\right] \\
= & -\frac{\phi_{B}}{2 \pi a \sin \theta} \frac{\mathrm{d}}{\mathrm{d} \varphi} \varphi \\
= & -\frac{\phi_{B}}{2 \pi a \sin \theta} .
\end{aligned}
$$

Eq. (27) indicates that the electric field and surface current density at the surface of the shell are only in the azimuthal direction, as expected from Eq. (1). The length of an azimuthal circle at the polar angle $\theta$ along the surface of the shell is given by 
$2 \pi a \sin \theta$. Eq. (28) indicates that $E_{\varphi}(a, \theta, \varphi)$ at each polar angle $\theta$ is given by the total electromotive force $\phi_{B}$ over the length of the corresponding circuit at the polar angle $\theta$, as expected. By Ohm's law the same inverse proportionality with $\sin \theta$ will be valid for the surface current density.

The surface charge distributions outside and inside the shell are related to the electric field through Gauss' law:

$$
\begin{aligned}
\sigma_{o}(a, \theta, \varphi)= & \lim _{r \rightarrow a} \varepsilon_{0} \vec{E}_{o}(r, \theta, \varphi) \cdot \hat{r} \\
= & \varepsilon_{0}\left\{\frac{\phi_{A}}{a}+\frac{\phi_{B}}{2 \pi a} \sum_{p=1}^{\infty}\left[\sum_{q=1}^{p} \frac{(-1)^{q-1}}{q}(p+1)(2 p+1)\right.\right. \\
& \left.\left.\times \frac{(p-q) !}{(p+q) !} I_{p q} P_{p}^{q}(\cos \theta) \sin (q \varphi)\right]\right\}
\end{aligned}
$$

$$
\begin{aligned}
\sigma_{i}(a, \theta, \varphi)= & -\lim _{r \rightarrow a} \varepsilon_{0} \vec{E}_{i}(r, \theta, \varphi) \cdot \hat{r} \\
= & \varepsilon_{0} \frac{\phi_{B}}{2 \pi a}\left[\sum_{p=1}^{\infty} \sum_{q=1}^{p} \frac{(-1)^{q-1}}{q} p(2 p+1)\right. \\
& \left.\times \frac{(p-q) !}{(p+q) !} I_{p q} P_{p}^{q}(\cos \theta) \sin (q \varphi)\right] .
\end{aligned}
$$

The total surface charge density is the sum of these two expressions, namely:

$\sigma_{t}=\sigma_{o}+\sigma_{i}$.

In Fig. 3 we plot the equipotentials, Eqs. (15) and (18), in the plane $z=0$ of the spherical shell with $\phi_{A}=0$ (no net charge in the shell). The current is in the clockwise direction, the bold circle represents the shell. The electric field lines which are perpendicular to these equipotentials are also contained in the plane $z=0$. This can be seen noting that for $\theta=\pi / 2 \mathrm{rad}$ we have $P_{p}^{q^{\prime}}(\cos \theta)=0$ for $p+q$ even (see [15, p. 733] combined with the recurrence relation (12.87) of [15]). Using the property that $I_{p q}$ is null for $p+q$ odd, we have that $E_{\theta}=0$ for both $r<a$ and $r>a$.

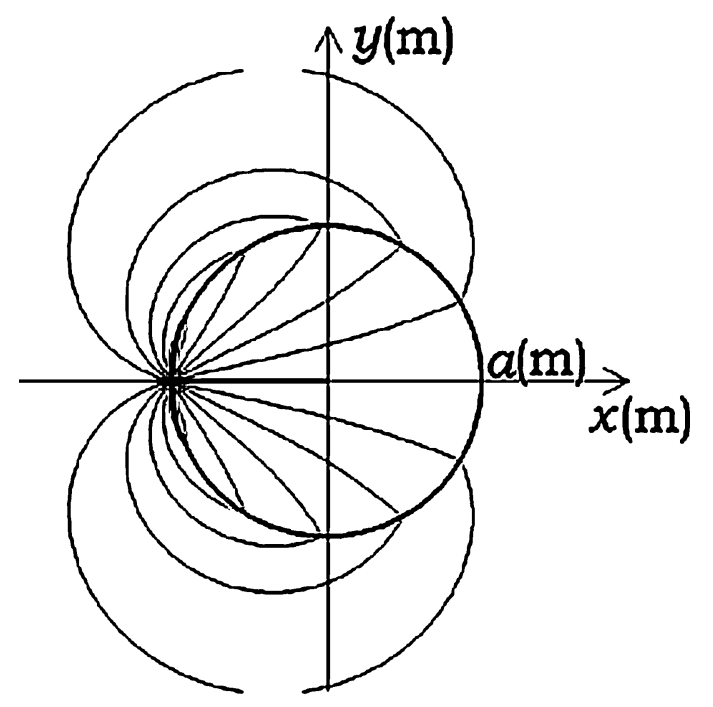

Fig. 3. Equipotentials in the plane $z=0$ obtained from Eqs. (15) and (18). The resistive spherical shell carries a clockwise steady current. The bold circle represents the shell. The projection of the battery is represented by the bold straight line going from $x=-a$ to $x=0$. The electric field has no $z$ component so the electric field lines are orthogonal to the equipotentials in this plane.

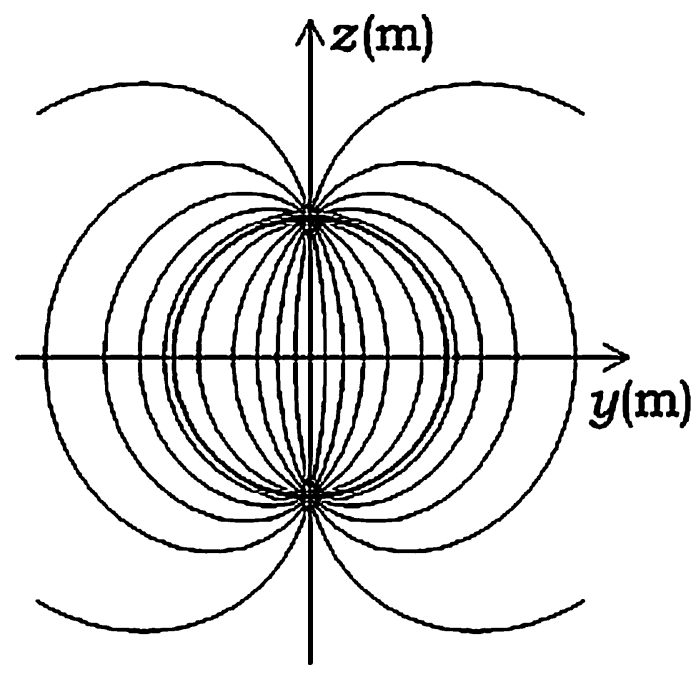

Fig. 4. Equipotentials in the plane $x=0$ of the spherical shell with $\phi_{A}=0$. The bold circle represents the shell. The current enters the plane of the paper at the left side of the circle and leaves the paper at the right side. We have used Eqs. (15) and (18).

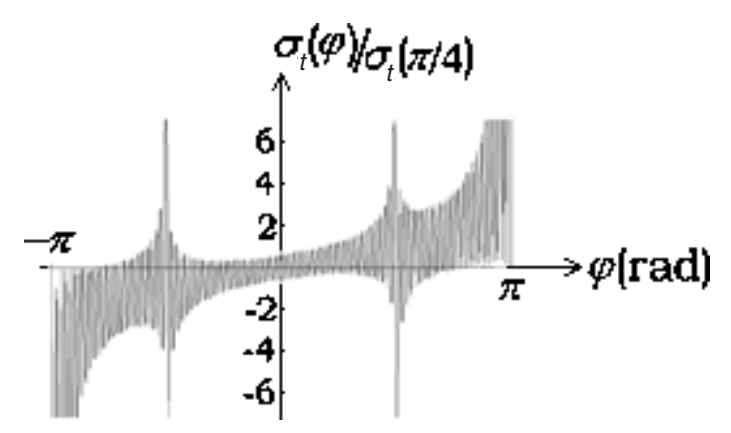

Fig. 5. Total surface charge density $\sigma_{t}(\varphi)$ as a function of the azimuthal angle $\varphi$ in the equatorial plane $z=0$ of a resistive spherical shell carrying a steady azimuthal current, normalized by its value at $\varphi=\pi / 4 \mathrm{rad}$. We utilized Eqs. (29) to (31) with the summation in $p$ going from $p=1$ to $p=100$.

In Fig. 4 we plot the equipotentials in the plane $x=0$ obtained from Eqs. (15) and (18). The current is entering the plane of the paper at the left side of the bold circle and leaving the plane of the paper at the right side. We utilized $\phi_{A}=0$. In this case the electric field lines are not contained in this plane $\left(E_{\varphi}\right.$ or $E_{x}$ are not null in the entire plane).

In Fig. 5 we plot the total surface charge density $\sigma_{t}$ in the equatorial plane as a function of the azimuthal angle $\varphi$, normalized by the value of $\sigma_{t}$ at $\varphi=\pi / 4 \mathrm{rad}$. The presence of the term $(-1)^{q} \sin (q \varphi)$ in Eqs. (29) and (30) causes a rapid variation in the numerical calculation of $\sigma_{t}$. This can be seen in the oscillation of Fig. 5.

The same effect can be observed in Heald's analysis of an infinite resistive cylindrical shell of radius $a$ carrying a steady azimuthal current, [7]. Beginning with a potential at the surface of the shell given by Eq. (1) with $\phi_{A}=0$ he obtained the total surface charge density along the shell as given by:

$\sigma_{t}^{\text {cylinder }}(a, \varphi, z)=\frac{\varepsilon_{0} \phi_{B}}{\pi a} \tan \frac{\varphi}{2}$.

This solution expanded in Fourier series is given by:

$\sigma_{t}^{\text {cylinder }}(a, \varphi, z)=\frac{2 \varepsilon_{0} \phi_{B}}{\pi a} \sum_{q=1}^{\infty}(-1)^{q-1} \sin (q \varphi)$ 


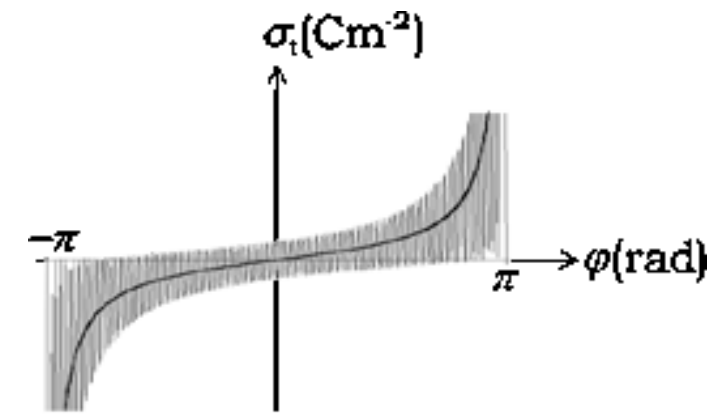

Fig. 6. Total surface charge density $\sigma_{t}$ of an infinite resistive cylindrical shell carrying a steady azimuthal current as a function of the angle $\varphi$, [7]. The bold line is a plot of the closed form solution of $\sigma_{t}(\varphi)$, Eq. (32), while the oscillatory line is a plot of $\sigma_{t}(\varphi)$ expressed in a Fourier series, Eq. (33).

In Fig. 6 we plot these two expressions. The bold line is given by Eq. (32), while the oscillating curve is a plot of Eq. (33) including 100 terms of the summation. Increasing the number of terms does not improve significantly the plot nor decrease the amplitude of oscillation around each value of $\varphi$. One way of smoothing out this oscillation is to consider at each angle $\varphi_{i}$ the average value of $\sigma_{t}$, $\overline{\sigma_{t}\left(\varphi_{i}\right)}$, namely:

$\overline{\sigma_{t}\left(\varphi_{i}\right)} \equiv \frac{1}{\Delta \varphi} \int_{\varphi_{i}-\Delta \varphi / 2}^{\varphi_{i}+\Delta \varphi / 2} \sigma_{t}(\varphi) \mathrm{d} \varphi$.

Fig. 7 is a plot of Eq. (32) overlaid on a plot of $\overline{\sigma_{t}\left(\varphi_{i}\right)}$ obtained from Eqs. (33) and (34). In this case we considered a whole oscillation of $\sigma_{t}\left(\varphi_{i}\right)$ around each angle $\varphi_{i}$. The plots coincided with one another (the two curves are indistinguishable in Fig. 7), indicating the correctness of this averaging procedure.

The oscillations on the plot of $\sigma_{t}(\varphi)$ shown in Fig. 5 probably occur because $\sigma_{t}$ is proportional to the radial component of the electric field that comes from differentiating a Fourier series, Eqs. (15) and (18). And sometimes there are convergence problems with the differentiation of Fourier series, [15, Section 14.4]. By raising the number of terms in the Fourier series of $\sigma_{t}$, Eq. (31), we increase only the number of oscillations in the curve. Neither the wrapping of the oscillation nor the wiggles at $\pm \pi / 2$ rad change significantly with a greater number of terms. We also investigated if these oscillations might arise from the numerical calculation of the coefficients $I_{p q}$ given by Eq. (14). The first approach was to utilize the associated Legendre

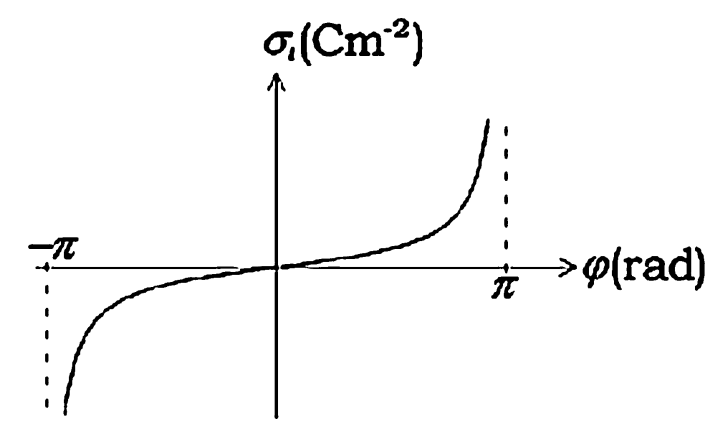

Fig. 7. Total surface charge density $\sigma$ of Heald, [7]. The summation that appears as an oscillation in Fig. 6, given by Eq. (33), is smoothed out by taking the mean value for each point of its surroundings (in this case, a whole oscillation around each point), utilizing Eq. (34). The closed analytical form, Eq. (32), is overlaid on it. Both plots coincided with one another, indicating the correctness of this averaging procedure.

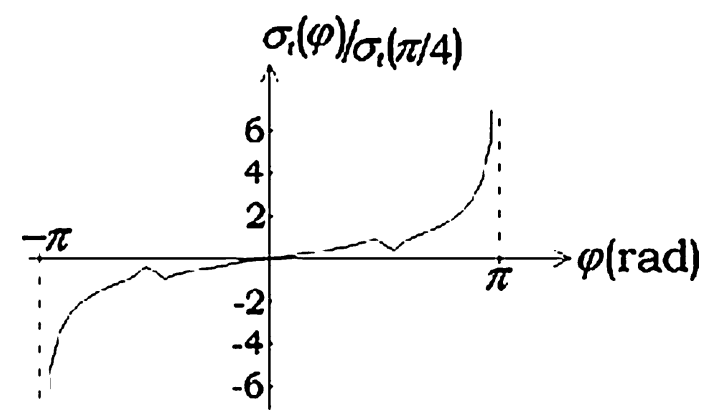

Fig. 8. Smoothed out plot of Fig. 5. We have used Eqs. (31) and (34).

functions directly to evaluate these definite integrals. The second approach was to utilize the recurrence formulas for these functions as developed by Leung, [16], with the initial values evaluated analytically. Although this last approach made the numerical calculations much faster than the first one, the final results and oscillations were the same in both cases.

We did not succeed in putting the series solutions given by Eqs. (29) and (30) in closed analytical forms. But utilizing the averaging procedure above yielded Fig. 8 . The wiggles around $\varphi= \pm \pi / 2 \mathrm{rad}$ should be due to the numerical approximation methods mentioned in the previous paragraph. These wiggles should not have any real physical significance, arising only from the mathematical treatment of the problem, although we were not able to reduce the size of the wiggles below what was shown in Fig. 8. The real curve should be smooth like Fig. 7. Fig. 8 indicates that $\sigma_{t}(\varphi)$ is linear with $\varphi$ far from the battery (i.e., around $\varphi=0 \mathrm{rad}$ ), diverging close to it (when $\varphi \rightarrow \pm \pi \mathrm{rad}$ ). This is the important physical result.

It should be noted that the resistivity of the material and the surface current involved in the problem are not explicitly mentioned in this treatment. However, we used that the conductor is ohmic, so that Ohm's law applies. That is, $\Delta \phi=R I$, where $\Delta \phi$ is the potential difference, $R$ is the resistance and $I$ is the current. Given the potential difference $\Delta \phi=\phi_{B}$ between the terminals of the battery and using the microscopic Ohm's law, $\vec{J}=\vec{E} / \rho_{R}$, where $\vec{J}$ is the current density and $\rho_{R}$ is the resistivity of the material, we have that the current density $\vec{J}$ is inversely proportional to the resistivity $\rho_{R}$, while $\vec{E}$ and the surface charge densities $\sigma_{o}$ and $\sigma_{i}$ are independent of this resistivity.

\section{Conclusion}

We obtained the total surface charge density, $\sigma_{t}$, potential, $\phi$, and electric field, $\vec{E}$, outside and inside a resistive spherical shell carrying a steady azimuthal current. We plotted the total surface charge density $\sigma_{t}$ as a function of the azimuthal angle $\varphi$. We obtained that $\sigma_{t}$ is linear with $\varphi$ far from the battery, diverging to infinity close to it. At great distances from the spherical shell the potential is that of a point charge plus that of an electric dipole, Eq. (17). The total charge $q$ and dipole moment $\vec{p}$ of this system is given by Eq. (17). Alternatively, they can also be found by $q=\iint \sigma_{t} \mathrm{~d} a$ and $\vec{p}=\iint \sigma_{t} \vec{r} \mathrm{~d} a$, where $\mathrm{d} a$ is an area element and the integration is over the surface of the system. Both approaches agree with one another, as expected.

\section{Acknowledgements}

The authors thank Faep/Unicamp and CNPq (Brazil) for financial support. 


\section{References}

1. Sommerfeld, A., "Electrodynamics", (Academic Press, New York, 1964).

2. Jefimenko, O. D., "Electricity and Magnetism", (Electret Scientific Company, Star City, 1989), 2nd ed.

3. Jackson, J. D., Am. J. Phys. 64, 855 (1996).

4. Assis, A. K. T. and Cisneros, J. I., in "Open Questions in Relativistic Physics", (edited by F. Selleri), (Apeiron, Montreal, 1998), pp. 177-185.

5. Griffiths, D. J., "Introduction to Electrodynamics", (Prentice Hall, New Jersery, 1999), 3rd ed.

6. Assis, A. K. T. and Cisneros, J. I., IEEE Trans. Circ. Sys. I 47, 63 (2000).

7. Heald, M. A., Am. J. Phys. 52, 522 (1984).

8. Stratton, J. A., "Electromagnetic Theory", (McGraw-Hill, New York, 1941).
9. Assis, A. K. T. and Mania, A. J., Rev. Bras. Ens. Fís. 21, 469 (1999).

10. Assis, A. K. T., Rodrigues, W. A. Jr. and Mania, A. J., Found. Phys. 29, 729 (1999).

11. Assis, A. K. T., Hernandes, J. A. and Lamesa, J. E., Found. Phys. 31, 1501 (2001).

12. Hernandes, J. A. and Assis, A. K. T., Am. J. Phys. 71, 938 (2003).

13. Hernandes, J. A. and Assis, A. K. T., Phys. Rev. E 68, 046611 (2003).

14. Moon, P. and Spencer, D. E., "Field Theory Handbook", (Springer-Verlag, Berlin, 1988), 2nd ed.

15. Arfken, G. B. and Weber, H. J., "Mathematical Methods for Physicists", (Academic Press, San Diego, 1995), 4th ed.

16. Leung, K. W., IEEE Trans. Antennas Propagat. 48, 961 (2000).

17. Jackson, J. D., "Classical Elecrodynamics", (John Wiley, New York, 1975), 2nd ed. 